\title{
Levator ani spasm: what to do after biofeedback fails
}

\author{
Jeremy Meyer ${ }^{1} \cdot$ Emilie Liot $^{1} \cdot$ Nicolas Christian Buchs ${ }^{1} \cdot$ Bruno Roche $^{1,2} \cdot$ Frédéric Ris $^{1}$
}

Received: 13 March 2020 / Accepted: 16 March 2020 / Published online: 10 April 2020

(c) Springer Nature Switzerland AG 2020

Nugent et al. performed an interesting single-center retrospective cohort study [1] regarding a proctologic condition leading to an important reduction in quality of life but still not well-known: the levator ani syndrome (LAS).

The authors included patients refractory to medical treatment who benefited from either injection of 200 UI of botulinum toxin into the levator ani identified by digital examination under general anaesthesia, or electro-galvanic stimulation with an Electro Galvanic Stimulator from Electro-Med Health Industries, USA, set with a $80 / \mathrm{min}$ pulse frequency, and progressive increase of voltage to reach 150-250 V for 30-45 min, usually in three sessions. The outcome was defined as subjective improvement or not, reported by the patient at $\leq 3$ months (short-term outcome) and $>9$ months (long-term outcome). The authors reported that Botulinum toxin injection led to greater improvement of LAS than electro-galvanic stimulation in the short term (71\% versus $21.4 \%, p=0.002$ ) but not in the long term ( $14.5 \%$ versus $7 \%, p=0.2$ ), with disappointing long-term results.

However, the study, despite being one of the few in the field, has several design flaws that limit interpretation of the findings.

First, the allocation of treatment was not randomized due to the retrospective nature of the study, with 76 patients in the botulinum toxin injection group in the definitive analysis versus 14 patients in the electro-galvanic stimulation group, which can lead to selection bias. As a consequence of that, there is an important heterogeneity in the groups: almost all patients with levator ani spasm in addition to pain were allocated to the botulinum toxin group, as these patients might

Frédéric Ris

Frederic.ris@hcuge.ch

1 Proctology Unit, Division of Digestive Surgery, University Hospitals of Geneva, Rue Gabrielle-Perret-Gentil 4, 1211 Genève 14 , Switzerland

2 Clinique de Grangettes, 7 chemin des Grangettes, 1224 Chêne-Bougeries, Switzerland be more prone to respond to a treatment aiming at relaxing the muscle. This constitutes a selection bias.

Second, the authors did not document if the patients received concomitant pelvic floor rehabilitation, which might alter the effect of the assessed treatment.

Third, there is a lack of documentation on exclusion and on patients lost to follow-up (both short-term and longterm). The proportion of positive responders might therefore be higher than the one reported by the authors.

Fourth, the injection of botulinum toxin was not performed under ultrasound guidance, therefore the target muscle might have been missed.

Fifth, the reported effect on striated muscle of botulinum toxin is 3-4 months. Therefore, this could explain the disappointing long-term effect observed at 9 month after the last injection. Indeed, as in anismus, the therapy should have been repeated every 3-4 months, at least twice, to hope for a sustainable effect. Of note, it has been reported that botulinum toxin injection can be safely repeated for years [2]. Further, in the long term, botulinum toxin injection might be more efficient in terms of cost-benefit ratio than electrogalvanic stimulation, and this also needs to be investigated.

From our point of view, the study by Nugent et al. adds information to an interesting field of research on a subject regarding which there is poor scientific evidence. The observation that botulinum toxin injection leads to shortterm improvement in $71 \%$ of patients suffering from LAS when compared to $21.4 \%$ for electro-galvanic stimulation $(p=0.002)$ indicates that botulinum toxin injection is a promising treatment. However, the study suffers from the important limitations listed above. We believe that therapy for refractory LAS deserves a well-conducted randomized controlled trial evaluating the different treatments in the field, including botulinum toxin injection and electro-galvanic stimulation, with comparison to placebo (with for example injection of saline solution or insertion of an ultrasound probe), with an appropriate sample size, to evaluate if these treatments, which are costly, time-consuming and also sometimes performed under general anaesthesia, offer any advantage over placebo or not. Further, the main outcome 
should be precisely defined and measured, if possible using objective instruments (visual analogical scale, measure of rectal balloon expulsion test, etc.), with attention given to duration of efficiency of the treatment.

Finally a cost-benefit analysis, evaluating the direct effects of treatment on relief of symptoms, but also including socio-economical aspects, should be conducted, as botulinum toxin injection in some healthcare systems botulinum toxin injections are not eligible for reimbursement.

To our knowledge, the only randomized controlled trial evaluating botulinum toxin for LAS was underpowered [3]. and observational studies report encouraging results for that therapy [4].

In addition, a randomized controlled trial has already demonstrated that biofeedback was superior to electrogalvanic stimulation, which was not different from digital massage in the overall cohort, for LAS [5]

We believe that botulinum toxin injection constitutes a promising therapy for LAS, as the procedure can also be performed under local anaesthesia, can be safely repeated over time, and, according to Nugent et al. leads to promising short-term improvement of LAS.

Funding None.

\section{Compliance with ethical standards}

Conflict of interest The authors disclose no conflict of interest.
Ethical approval This article does not contain any studies with human participants or animals performed by any of the authors.

Informed consent For this type of study, formal consent is not required.

\section{References}

1. Nugent E, Beal M, Sun G et al (2019) Botulinum toxin A versus electrogalvanic stimulation for levator ani syndrome: is one a more effective therapy? Tech Coloproctol. https://doi.org/10.1007/ s10151-019-02103-

2. Mejia NI, Vuong KD, Jankovic J (2005) Long-term botulinum toxin efficacy, safety, and immunogenicity. Mov Disord 20:592-597

3. Rao SS, Paulson J, Mata M et al (2009) Clinical trial: effects of botulinum toxin on Levator ani syndrome-a double-blind, placebo-controlled study. Aliment Pharmacol Ther 29:985-991

4. Ooijevaar RE, Felt-Bersma RJF, Han-Geurts IJ et al (2019) Botox treatment in patients with chronic functional anorectal pain: experiences of a tertiary referral proctology clinic. Tech Coloproctol 23:239-244

5. Chiarioni G, Nardo A, Vantini I et al (2010) Biofeedback is superior to electrogalvanic stimulation and massage for treatment of levator ani syndrome. Gastroenterology 138:1321-1329

Publisher's Note Springer Nature remains neutral with regard to jurisdictional claims in published maps and institutional affiliations. 